\title{
El entrenador de alto nivel en triatlón: entorno próximo y cualidades fundamentales para el rendimiento

High level triathlon coach: close environment and basic
performance qualities

\author{
Germán Ruiz Tendero \\ Universidad Complutense de Madrid \\ Juan José Salinero Martín \\ Universidad Camilo José Cela
}

\begin{abstract}
Resumen
La figura del entrenador de alto nivel (E.A.N.) en triatlón y su entorno apenas ha sido estudiada, y sin embargo puede estar ejerciendo una gran influencia sobre el microsistema deportivo. Estudiar este microsistema es el objetivo del presente estudio en función de los siguientes factores: interacción con sus deportistas, objetivos deportivos, grado de satisfacción con diferentes agentes del entorno (triatletas, otros entrenadores, federaciones y club), ámbito laboral, nivel académico y entorno familiar. Además, se pretende conocer qué cualidades atribuibles a un buen entrenador de alto nivel en triatlón son las mejores valoradas por los propios entrenadores. En este estudio participan 14 entrenadores españoles de alto nivel en activo (uno menos que el universo de la muestra objeto). Todos ellos hombres (edad media $=37,43$ años $\pm 7,27$ ). El diseño de investigación descriptivo-cuantitativo utiliza el cuestionario como instrumento. El cuestionario fue pasado en periodo no competitivo por el mismo investigador mediante entrevistas personales mayoritariamente, o de forma telemática en su defecto. Los resultados muestran que el triatlón de alto nivel español se rige por un sistema centralizado en centros de rendimiento. La presencia del entrenador en las competiciones (sólo uno acudía a todas) no supone un factor determinante del rendimiento. El ratio número de atletas de alto nivel por entrenador es de $3,71( \pm 3,04)$ y el ratio total contando con otros triatletas de diferente nivel es de $8( \pm 4,78)$, adecuado para las condiciones actuales pero mejorable en el alto nivel. El nivel académico de los E.A.N. es muy bueno y específico a su labor deportiva. La cualidad confianza para el diálogo personal (4,69 sobre 5) resulta ser la más valorada por parte de los propios entrenadores, anteponiendo el plano personal al deportivo.
\end{abstract}

Palabras clave: triatlón de alto nivel; entrenador; factores de rendimiento.

\begin{abstract}
Not much attention has been paid to the high level coach (H.L.C.) in triathlon and his environment; howeve this one could be influencing the sport microsystem. The aim of the present study is to describe this microsystem. The assessed factors are: interaction coach-athletes, sports goals, satisfaction with various environmental agents (triathletes, other coaches, federations and club), workplace, academic level and family environment. In addition, a number of qualities associated with a successful coaching are valued. Participants are 14 H.L.C. on active service out of a universal sample of 15 in high level Spanish triathlon. All of them are males (average age $=37,43$ years old $\pm 7,27$ ). Using the questionnaire, the research follows a quantitative and descriptive design. The personal interviews were carried by the same researcher in the preparatory period. The results show Spanish high level triathlon as a sports system centralized in centers of performance for triathletes. Triathlon nature and coaches' environment do not make their presence in the competition be determinant in athlete's performance (only one coach was present in all competitions). The ratio number of elite athletes / coach is $3.71( \pm 3.04)$, and total count ratio between athletes of different levels is $8( \pm 4.78)$. This is a suitable number for current conditions, but it could be improvable in high level conditions. Coaches' academic level is very good and it is specific to their profession. Confidence in personal interaction $(4,69$ over 5$)$ proves to be the most valued factor by the H.L.C. In this sense, they consider the personal sphere to be more relevant than sports sphere.
\end{abstract}

Key words: high level triathlon; coach; performance factors.

Correspondencia/correspondence: Germán Ruiz Tendero

C/ Rector Royo Villanova s/n. Facultad de Educación (UCM). 28040 - Madrid (España).

E-mail: g.germanruiz@gmail.com 
Ruiz, G.; Salinero, J. J. (2011) El entrenador de alto nivel en triatlón: entorno próximo y cualidades fundamentales para el rendimiento. Revista Internacional de Ciencias del Deporte, 23(7), 113-125. http://www.cafyd.com/REVISTA/02304.pdf

\section{Introducción}

$\mathrm{E}$ triatlón, deporte olímpico cada vez más conocido, ha sido objeto de numerosas investigaciones en las que, sin embargo, pasan desapercibidos numerosos factores que pueden estar determinando el éxito deportivo. Desde los años 80 la investigación en triatlón se ha centrado en estudiar las variables fisiológicas bajo diferentes condiciones (Farber, 1987; Kreider, 1988; Miura, 1999; Zhou; Robson; King, y Davie, 1997) y parámetros de entrenamiento (Baker y Deakin, 2003; Banister; Carter, y Zarkadas, 1999; Hue; Valluet; Blonc, y Hertogh, 2002; O'Toole, 1989). Aspectos como la personalidad y el entorno, tanto del deportista como del entrenador, han pasado más desapercibidos en el ámbito de la investigación de este deporte. Sin embargo, y al amparo del marco teórico que ofrece la Teoría de Sistemas (Bertalanffy, 1968), en la actualidad el deportista y su rendimiento no puede ser entendido sin una concepción integral de él mismo y de su entorno. Rossi (1977) y Tatano (1977) fueron pioneros en la utilización del término "sistema deportivo", que ha venido siendo desarrollado para una mejor comprensión de un fenómeno vivo, cambiante y global como es el deporte (De Bosscher y De Knop, 2002; Digel, 2002; Sánchez-Bañuelos, 2002)

Dentro de este sistema deportivo global, se localizan al deportista y su entrenador en lo que se ha venido denominando el "microsistema" o entorno próximo del deportista (club deportivo, familia). Los estudios de microsistema en triatlón son realmente escasos y tienden a ser más de corte psico-social, como el trabajo de Hilliard (1986) acerca de la representación de las emociones en deportistas de resistencia de larga duración. Más actual es el estudio de Chang y Johnson (1995) sobre las motivaciones competitivas en triatlón.

El rendimiento del deportista difícilmente puede percibirse como un logro exclusivamente personal; incluso en los deportes individuales puede hablarse de trabajo en equipo. Este rendimiento es visto como el resultado de la interacción entre el atleta, el entrenador y el entorno de entrenamiento (Cavalheiro; Soter, y Gagliardi, 2005; Haslam y Smith, 2001). Otra forma de apreciar esta combinación, más cercana a nuestro estudio, es desde el alto rendimiento, en el que intervendrían el joven talento, el entrenador experto y un tiempo y facilidades adecuados (Broom, 1981). Conocer el contexto en el que se desarrolla la labor del E.A.N. nos va a permitir en un futuro establecer un camino óptimo para el desarrollo y mejora de las condiciones en las cuales tiene lugar el proceso del entrenamiento deportivo. Al respecto, Sage (1980) considera que las experiencias de socialización llevan a predicciones sobre creencias, comportamientos y orientación de valores de los entrenadores, valiéndose para la discusión de datos empíricos. Y es en esa orientación de valores donde, a igual formación sobre entrenamiento, puede radicar la clave del buen entrenador. Algunas de estas circunstancias ya han sido estudiadas en la literatura como las percepciones del entrenamiento y la competición (Côte; Salmela, y Russell, 1995), las tareas organizativas (Côte y Salmela, 1996), las habilidades de comunicación (G. A. Bloom; Schinke, y Salmela, 1998) o el proceso de dirección y tutela del deportista (Bloom; Durand-Bush; Schinke, y Salmela, 1998). Se ha documentado por ejemplo que los entrenadores expertos valoran como importante la comprensión de los sentimientos de sus atletas (Bloom y Salmela, 2000).

En el ámbito del triatlón no se dispone de información respecto a los entrenadores, si no es en artículos de revistas de divulgación que, por lo general, hacen referencia a entrevistas personales.

En este estudio se plantean dos objetivos:

1) Describir el entorno próximo del entrenador de alto nivel teniendo en cuenta los siguientes factores: interacción con sus deportistas, objetivos deportivos, grado de satisfacción con 
Ruiz, G.; Salinero, J. J. (2011) El entrenador de alto nivel en triatlón: entorno próximo y cualidades fundamentales para el rendimiento. Revista Internacional de Ciencias del Deporte, 23(7), 113-125. http://www.cafyd.com/REVISTA/02304.pdf

diferentes agentes del entorno (triatletas, otros entrenadores, federaciones y club), ámbito laboral, nivel académico y entorno familiar.

2) Conocer qué cualidades atribuibles a un buen entrenador de alto nivel en triatlón son las mejores valoradas por los propios entrenadores.

\section{Método}

El presente estudio se desarrolla siguiendo un diseño descriptivo de corte cuantitativo del tipo encuesta, siguiendo las propuestas metodológicas para el área de las Ciencias Sociales (Alvira, 2004; Cea, 1999; García; Ibáñez, y Alvira, 2002). El estudio se completa con pruebas de comparación entre grupos.

\section{Participantes}

El universo de la muestra en España estaba compuesto por 15 entrenadores de alto nivel (E.A.N.) en triatlón, de los cuales sólo uno no pudo participar en el estudio. Por lo tanto la muestra se compone de 14 entrenadores, todos ellos hombres (edad media= 37,43 años $\pm 7,27)$. El requisito para formar parte de la muestra de entrenadores, y por tanto pertenecer al grupo E.A.N., fue estar entrenando en el momento del estudio a algún triatleta que hubiera formado parte en alguna ocasión de la selección española de triatlón participando en competiciones de ámbito internacional (Juegos Olímpicos, Campeonatos del Mundo, Copas del Mundo, Campeonatos de Europa). Para la selección de los triatletas de alto nivel, y por tanto de sus entrenadores, consultamos la base de datos del Instituto de Ciencias Aplicadas al Entrenamiento de Leipzig (IAT) (Institut für Angewandte Trainingswissenschaft of Leipzig), en la que aparecen recogidos todos los resultados internacionales desde el año 1989 con diversas opciones de clasificación y búsqueda. Otra base de datos revisada para la triangulación fue la del CSD (Consejo Superior de Deportes), en la que aparecen aquellos triatletas considerados como deportistas de alto nivel, con o sin beneficios vigentes, también con diversas opciones de búsqueda.

\section{Instrumento}

Se utilizó el cuestionario como herramienta metodológica, teniendo en cuenta que es el instrumento más utilizado para la obtención de datos en estudios de sociología empírica (García-Ferrando, 2003). El cuestionario fue diseñado al efecto siguiendo 3 vías conexas: 1) la propuesta de los expertos que pasó por un brainstorming, para posteriormente definir las variables y agruparlas. Para el brainstorming se emplearon las dos fases propuestas por Osborn (1963) de creación y posterior evaluación en sendas sesiones de 35 minutos aproximadamente cada una, considerándose este un tiempo adecuado para esta metodología (Isaksen, 1998); 2) un estudio previo llevado a cabo con 60 triatletas de nivel competitivo a nivel regional (Ruiz; Salinero, y Sánchez-Balueños, 2008), y 3) la revisión bibliográfica sobre estudios sociológicos y cuestionarios relacionados con deportistas de élite en los cuales estuviera presente la figura el entrenador (García-Ferrando, 1996; Hill; McConnell; Forster, y Moore, 2002; J. Pérez, 1977). En una segunda fase, el instrumento fue revisado por 4 expertos para afianzar la validez del mismo: dos de ellos investigadores del ámbito de la sistemática deportiva, un tercer investigador con experiencia en triatlón y sistemas deportivos y una cuarta persona de la Federación Española de Triatlón (FETRI) en contacto directo con el cuerpo técnico. Tras las modificaciones oportunas, se pasó, en una tercera fase, un estudio piloto a los 48 triatletas dirigidos por los entrenadores que serían encuestados. Las preguntas para unos y otros fueron de similar orden. De esta forma reforzamos las cualidades que los deportistas esperan de un buen entrenador, para después preguntarles a éstos últimos. Finalmente el cuestionario, de seis páginas de extensión, quedó dividido en cinco secciones: 
Ruiz, G.; Salinero, J. J. (2011) El entrenador de alto nivel en triatlón: entorno próximo y cualidades fundamentales para el rendimiento. Revista Internacional de Ciencias del Deporte, 23(7), 113-125. http://www.cafyd.com/REVISTA/02304.pdf

carta de presentación, datos personales, entorno deportivo, entorno familiar y valoración profesional. Las otras cuatro secciones del cuestionario estaban compuestas en su mayor parte por preguntas de respuesta abierta (56,52\%), pero también mixta (26,08\%) y cerrada $(17,39 \%)$ de elección múltiple o dicotómica. En su conjunto, el cuestionario pretende valorar tres dimensiones: entorno deportivo, entorno familiar y valoración profesional, cuyas variables se especifican en la tabla 1. Para la valoración de la última dimensión, se recogieron en una tabla una serie de cualidades positivas atribuibles a un entrenador. Estas cualidades fueron puntuadas por los entrenadores a través de una escala de tipo Likert (1932) de cinco niveles (1=sin importancia; $2=$ poca importancia; $3=$ importancia moderada; $4=$ importante; $5=$ muy importante). Para la muestra analizada $(n=14)$ el modelo de consistencia interna de Cronbach utilizado en la dimensión "valoración profesional” mostró un valor de 0,627 (0,698 para elementos tipificados), lo cual supone una fiabilidad intermedia $(0,4-0,8)$. La consistencia obtuvo valores aún más aceptables cuando el ítem "interés por la innovación" fue suprimido (alfa=0,65 y 0,71 para elementos tipificados). El cuestionario fue implementado en periodo no competitivo de la temporada, mediante entrevista personal en la mayoría de los casos y llevado a cabo por un único investigador. Allí donde no fue posible contactar personalmente con los entrenadores, se llevó a cabo de forma autoadministrada telemáticamente.

Tabla 1. Dimensiones del cuestionario y sus respectivas variables.

\begin{tabular}{|l|l||}
\hline Dimensión & Variables \\
\hline Entorno deportivo & -Titulación deportiva y estudios. \\
& -Número y nivel de triatletas a los que entrena. \\
& -Interacción semanal con los triatletas a los que entrena. \\
& -Presencia en competiciones internacionales. \\
& -Objetivos a medio y largo plazo. \\
& -Grado de satisfacción y trato con diferentes agentes del entorno. \\
& -Ocupación laboral paralela. \\
\hline Entorno familiar & -Práctica deportiva de familiares directos. \\
\hline Valoración profesional & -Estatus socioeconómico. \\
\hline \hline
\end{tabular}

\section{Análisis de datos}

Los datos fueron codificados y analizados mediante el software SPSS v. 15.0. El análisis estadístico consistió, según la tipología de las variables, en extraer los estadísticos descriptivos, en aplicar la prueba chi-cuadrado de Pearson para las comparaciones de variables no paramétricas y la $\mathrm{r}$ de Pearson para las correlaciones entre variables.

\section{Resultados}

\section{Interacción del entrenador con los triatletas de alto nivel a los que entrena}

Como media, cada entrenador dirige a un total de 8 triatletas $( \pm 4.78)$, sin embargo la media de triatletas que compiten a nivel internacional dirigidos por un mismo entrenador es de 3.71 ( \pm 3.04$)$. Analizando estos datos en función del ámbito donde desempeña su labor el entrenador, obtenemos los resultados que se muestran en la figura 1. 
Ruiz, G.; Salinero, J. J. (2011) El entrenador de alto nivel en triatlón: entorno próximo y cualidades fundamentales para el rendimiento. Revista Internacional de Ciencias del Deporte, 23(7), 113-125. http://www.cafyd.com/REVISTA/02304.pdf

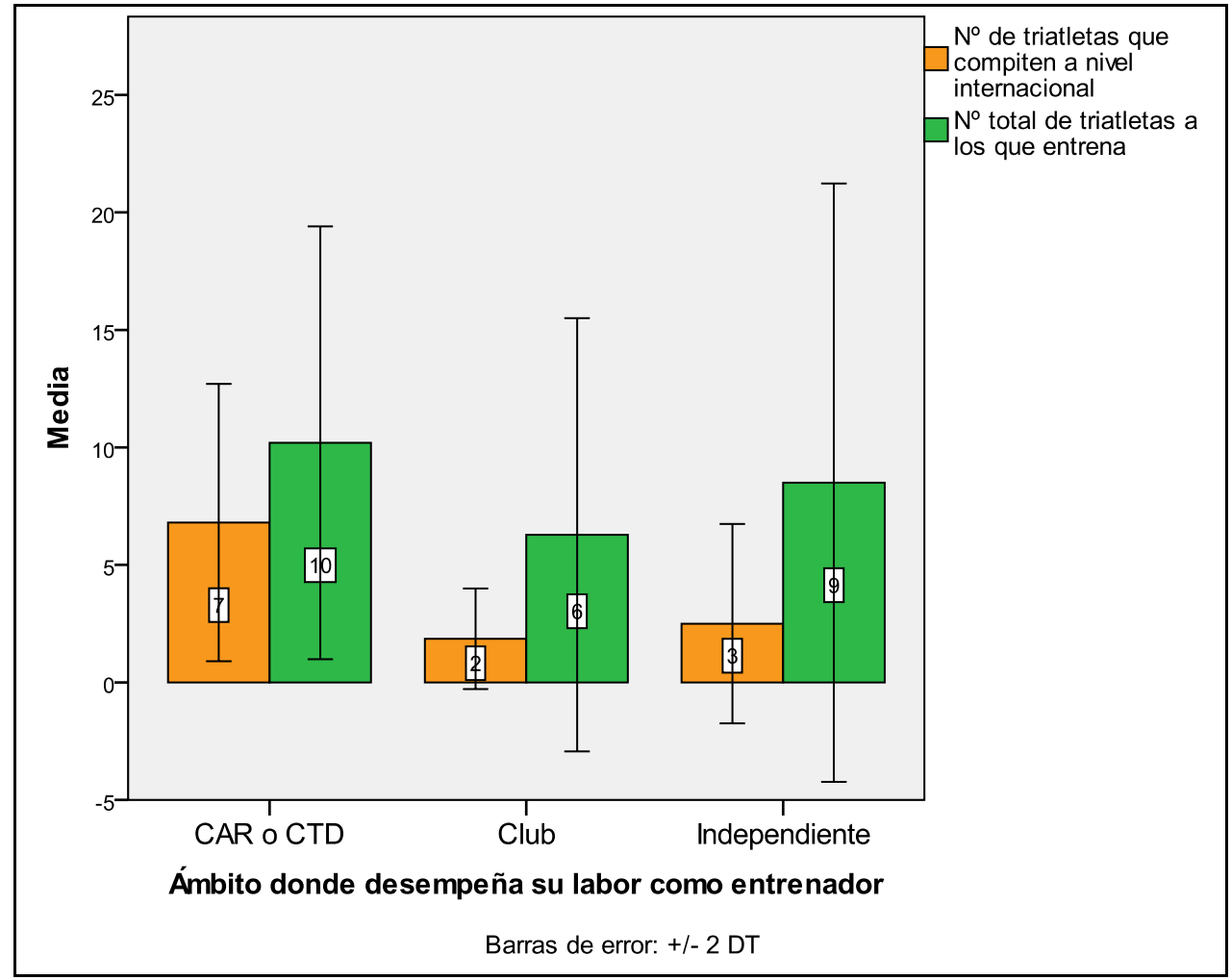

Figura 1. Número de triatletas que los entrenadores dirigen en función del ámbito donde desempeñan su labor.

Los entrenadores que desempeñan su labor en Centros de Alto Rendimiento (CAR) o Centros de Tecnificación Deportiva (CTD) dirigen significativamente a un mayor número de triatletas de nivel internacional $(\mathrm{F}=8,9 ; \mathrm{p}<, 01)$ que los entrenadores que trabajan en clubes.

La interacción personal semanal con los triatletas de más alto nivel suele ser continuo para la mayoría de los entrenadores (75\%). Para 3 entrenadores la interacción personal es esporádica. Si analizamos la interacción personal con los deportistas en función del ámbito donde el entrenador desempeña su labor (figura 2), apreciamos cómo aquellos entrenadores que se encuentran en un CAR o CTD tienen un contacto continuo con sus deportistas.

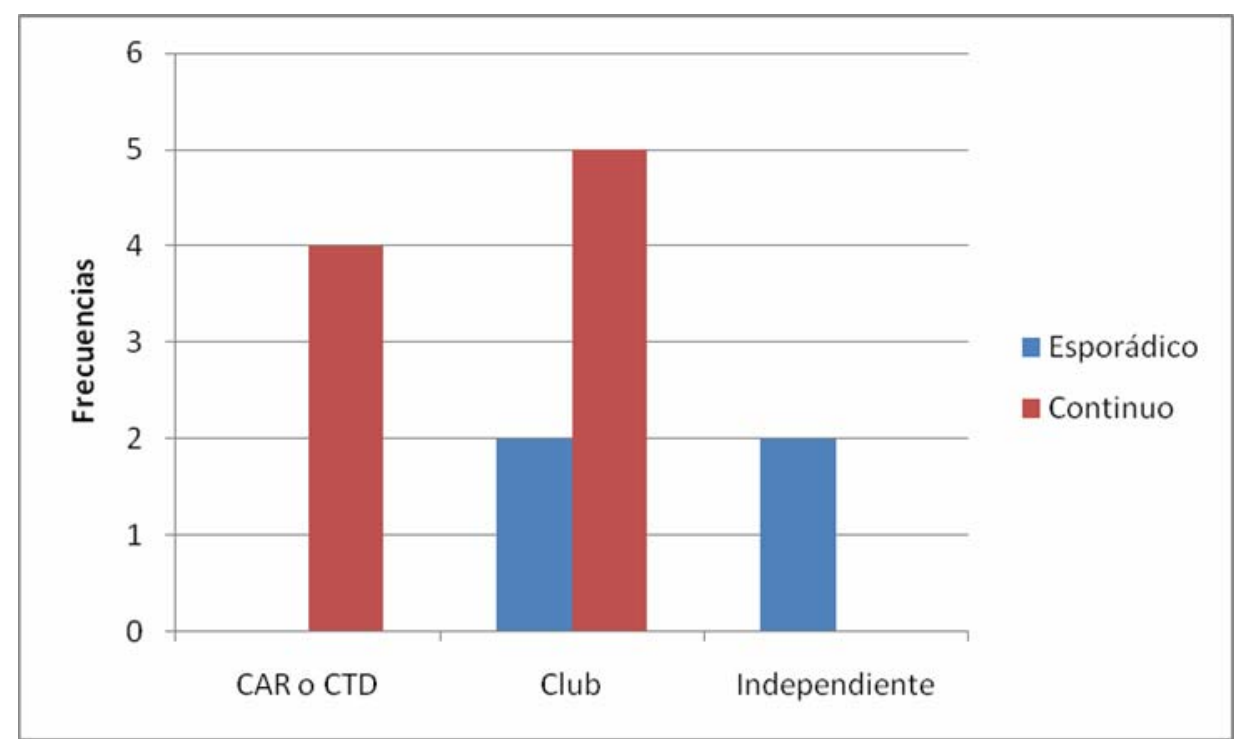

Figura 2. Frecuencia de la interacción personal del entrenador con sus triatletas según el ámbito donde desempeña su labor como entrenador. 
Ruiz, G.; Salinero, J. J. (2011) El entrenador de alto nivel en triatlón: entorno próximo y cualidades fundamentales para el rendimiento. Revista Internacional de Ciencias del Deporte, 23(7), 113-125. http://www.cafyd.com/REVISTA/02304.pdf

Ante la pregunta “¿Acompaña a sus deportistas en competiciones internacionales?”, las respuestas fueron variadas. Sólo uno afirmó ir siempre y dos de ellos acudían a casi todas. 3 entrenadores afirmaron ir sólo a las importantes. Las razones que aludían los entrenadores para no acudir con sus deportistas a estas competiciones fueron la imposibilidad económica y temporal, y la incompatibilidad con otros trabajos.

\section{Objetivos como entrenador}

Objetivos a medio plazo: La máxima importancia la adquieren los objetivos competitivos internacionales (77,8\%). Los otros dos objetivos que se han dado son sacar lo mejor de los deportistas (33,3\%) y crear una base en categoría de menores (11,1\%).

Objetivos a largo plazo: Los objetivos competitivos y la participación en los Juegos Olímpicos, son los objetivos principales para la mayoría de los entrenadores (63.7\%). Existe también un interés por parte de alguno de ellos de potenciar las categorías de menores y el llegar a generar un grupo estable de alto nivel.

\section{Grado de satisfacción y trato con diferentes agentes del entorno}

Las respuestas a este apartado se muestran en función de una escala Likert de nivel 5 para: muy malo, malo, sin trato, bueno y excelente.

Grado de satisfacción y trato con otros compañeros entrenadores: El trato con otros entrenadores es mayoritariamente bueno (61,5\%). El 23,1 \% respondió que no tiene trato, mientras que el 15,4\% afirmó que el trato era excelente.

Grado de satisfacción y trato con su club: El trato y la satisfacción con el club es calificado por la mayoría de los entrenadores como excelente (50\%) y bueno (33,3\%). El 16,7\% indicó que no tenía trato.

Grado de satisfacción y trato con sus triatletas de alto nivel: El grado de satisfacción con los triatletas obtienen la más alta valoración en cuanto a excelencia $(61,5 \%)$, sin que existan diferencias significativas $\left(\mathrm{X}^{2}=1,397\right.$, sig. $=$,382) entre entrenadores que tienen un trato continuo con sus deportistas y los que tienen un trato esporádico durante los entrenamientos. Esta valoración no baja de buena.

Grado de satisfacción y trato con las federaciones: Con la FETRI sucede algo parecido como con el caso de las Federaciones Regionales: el grado de satisfacción para la mayoría es bueno (57,1\%), sin embargo aparecen algunas valoraciones desfavorables $(14,2 \%)$.

Ámbito laboral y estudios

Estudios completados: Es muy significativo el hecho de que el 78,5\% de los E.A.N. encuestados posean la titulación de Licenciado en Ciencias de la Actividad Física y del Deporte (CAFD) y uno de ellos estudios de postgrado relacionados con las ciencias del deporte. El 14,3\% posee el Bachillerato y el 7,1\% Magisterio especialidad Educación Física.

Ocupaciones aparte del entrenamiento deportivo: Sólo el 16,7\% se dedican exclusivamente al entrenamiento deportivo. La gran mayoría $(83,4 \%)$ se encuentran trabajando aparte de su labor como entrenadores.

Tipo de trabajo al que se dedican: Todos los entrenadores que tienen un puesto de trabajo aparte del de entrenador, lo desempeñan en relación con la actividad física, tanto en el ámbito privado (50\%) como en el público (50\%). Estos puestos de trabajo son los especificados en la tabla 2. 
Ruiz, G.; Salinero, J. J. (2011) El entrenador de alto nivel en triatlón: entorno próximo y cualidades fundamentales para el rendimiento. Revista Internacional de Ciencias del Deporte, 23(7), 113-125. http://www.cafyd.com/REVISTA/02304.pdf

Tabla 2. Puesto de trabajo para aquellos entrenadores que no se dedican exclusivamente al entrenamiento en el alto nivel.

\begin{tabular}{|l|l|l|}
\hline Puesto de trabajo & N & $\%$ \\
\hline Director técnico y entrenador de un club de natación & 1 & 10 \\
\hline Entrenador/técnico deportivo & 2 & 20 \\
\hline Fisiólogo & 1 & 10 \\
\hline Gestor deportivo & 1 & 10 \\
\hline Profesor de Educación Física & 4 & 40 \\
\hline $\begin{array}{l}\text { Profesor universitario en Ciencias de la Actividad Física y del } \\
\text { Deporte }\end{array}$ & 1 & 10 \\
\hline Total & 10 & 100 \\
\hline
\end{tabular}

\section{Entorno familiar}

Estatus socioeconómico familiar estimado: Por regla general los entrenadores de triatlón en el alto rendimiento se sitúan dentro de la clase media (61,5\%). Les siguen clase media-alta (23,1\%) y media-baja $(15,4 \%)$.

Práctica deportiva de los familiares directos (padres, hermanos, pareja, hijos): De los 14 entrenadores, el 28,57\% afirman tener algún familiar que practica triatlón o alguna de las disciplinas de que se compone. Los familiares que practican deporte varían desde padres a hijos.

Importancia atribuida a las diferentes cualidades positivas de un entrenador (tabla 3)

La confianza para el diálogo personal $(4,69 \pm 0,48)$ se sitúa como el factor más valorado por los entrenadores, seguido por los conocimientos de entrenamiento y capacidad para motivar y animar. De cualquier forma, la valoración de los 9 primeros factores sigue una forma gradual, sin grandes bajadas. A todos estos factores se les concede gran importancia.

Tabla 3. Valoración, por parte de los entrenadores, de las diferentes cualidades atribuibles a un buen entrenador.

\begin{tabular}{|l|l|l|l|l||}
\hline $\begin{array}{l}\text { Orden de } \\
\text { importancia }\end{array}$ & Factores & Media & Moda & Desv. típ. \\
\hline 1 & Confianza para el diálogo personal & 4,69 & 5 &, 48 \\
\hline 2 & Conocimientos de entrenamiento & 4,62 & 5 &, 65 \\
\hline 2 & Capacidad para motivar y animar & 4,62 & 5 &, 87 \\
\hline 4 & Capacidad para transmitir & 4,54 & 5 &, 66 \\
\hline 5 & Personalidad & 4,46 & 5 &, 87 \\
\hline 6 & Conocimientos estratégicos del deporte & 4,31 & 5 & 1,2 \\
\hline 7 & Habilidad para organizar y dirigir & 4,23 & 4 &, 72 \\
\hline 8 & Apoyo en el establecimiento de metas & 4,15 & 4 &, 68 \\
\hline 9 & $\begin{array}{l}\text { Interés por la innovación aplicada al } \\
\text { entrenamiento }\end{array}$ & 4 &, 91 \\
\hline 10 & Competencia en las destrezas & 3,38 & 3 & 1,12 \\
\hline 11 & Presencia durante los entrenamientos & 3,15 & 3 & 1,06 \\
\hline 12 & Presencia durante las competiciones & 3,08 & 3 & 1,03 \\
\hline & & & \\
\hline
\end{tabular}


Ruiz, G.; Salinero, J. J. (2011) El entrenador de alto nivel en triatlón: entorno próximo y cualidades fundamentales para el rendimiento. Revista Internacional de Ciencias del Deporte, 23(7), 113-125. http://www.cafyd.com/REVISTA/02304.pdf

\section{Discusión}

El primer objetivo que se planteaba en esta investigación se refería al estudio del entorno próximo del entrenador de alto nivel. El primer factor analizado ha sido la interacción en el binomio deportista-entrenador. Al respecto, surge la cuestión de si podemos considerar adecuado un ratio número de atletas/entrenador de 3,71 $( \pm 3,04)$ en el alto nivel de un deporte individual, pero que sigue estrategias de equipo, como ocurre por ejemplo en el ciclismo. Sabiendo, además, que los entrenadores entrenan a la vez a otros triatletas de menor nivel. No disponemos de datos al respecto en la bibliografía que nos indiquen la adecuación de este dato, pero si analizamos la naturaleza del propio deporte, sabemos que la respuesta fisiológica está en estrecha relación con el rendimiento (Laursen, 2001; Sleivert y Rowlands, 1996; Sleivert y Wenger, 1993) y que cada triatleta responde a diferentes umbrales de entrenamiento (Díaz, et al., 2009), que a su vez son también diferentes para cada una de las especialidades en las que entrena: natación, ciclismo y carrera (Kohrt, Morgan, Bates y Skinner, 1987). Este hecho conlleva un gran esfuerzo por parte del entrenador en el proceso de planificación, que ha de ser ajustada a cada perfil individual. Siendo estrictos podemos considerar este ratio como mejorable, pero no así teniendo en cuenta el contexto deportivo y personal del entrenador, que ejerce su labor de forma vocacional en un deporte escasamente profesionalizado. Esta circunstancia lleva a la mayoría de los E.A.N. a tener otro trabajo aparte del de entrenador. Las únicas referencias específicas encontradas sobre el ratio número de atletas por entrenador, llamado en la terminología anglosajona coach to athlete ratio, las podemos ver en algunos organismos como la British Triathlon (2011) (Federación de Triatlón de Gran Bretaña), la cual establece un ratio de entrenador: triatleta de 1:15 para jóvenes deportistas entre 8-16 años, o 1:5 si la sesión de entrenamiento se produce en aguas abiertas. Estos son mínimos establecidos para garantizar la calidad y seguridad en las sesiones. Aunque estos datos se establecen para jóvenes en periodo de formación, los resultados hallados en el presente estudio mejoran con creces estos ratios.

Por otro lado, ha quedado patente que para lograr un buen rendimiento en triatlón de alto nivel no es necesaria la presencia del entrenador tanto en el entrenamiento como en las competiciones. Este dato objetivo coincide con que los propios entrenadores conceden el menor valor a la presencia del entrenador como factor determinante para el buen trabajo del mismo. No pretendemos, nada más lejos de la realidad, supeditar la figura del entrenador a labores exclusivas de planificación y control del entrenamiento. Uno de los entrenadores de este estudio, a cuyo cargo está un deportista de élite a nivel mundial, no reparaba en señalar la gran importancia que tiene la comunicación con el triatleta, el saber escucharle. Estos aspectos facilitan en gran medida la simbiosis deportista-entrenador, y hacen que éste llegue a comprender hasta qué punto el triatleta está o no asimilando el entrenamiento, siempre sujeto a posibles modificaciones en función de la respuesta del deportista (Varela, 2003). De hecho la cualidad del entrenador más valorada fue la confianza para el diálogo personal. Se lleve a la práctica o no, en este ámbito se es consciente de la importancia que tiene este aspecto en el día a día del entrenamiento.

Conocemos que los entrenadores vinculados a los CAR dirigen de forma significativa $(p<, 01)$ a un número mayor de triatletas de alto nivel, en comparación con los entrenadores que desarrollan su actividad en un club. Este dato nos sugiere que el sistema de alto nivel para el triatlón se centra fundamentalmente en centros de rendimiento específicos, tal y como se ha mostrado en otros estudios en este mismo ámbito (Ruiz, 2006). Los entrenadores que ejercen en CAR dirigen a una media de 7 triatletas de nivel internacional. Este tipo de direcciones "masivas" no tienen porqué funcionar mal si la implicación del deportista es correcta y existe todo un equipo de apoyo, como suele ocurrir en este tipo de centros subvencionados por el 
Ruiz, G.; Salinero, J. J. (2011) El entrenador de alto nivel en triatlón: entorno próximo y cualidades fundamentales para el rendimiento. Revista Internacional de Ciencias del Deporte, 23(7), 113-125. http://www.cafyd.com/REVISTA/02304.pdf

Estado, aunque toda situación es susceptible de ser mejorable. Lo que sí apreciamos es que todos los entrenadores que desempeñan su labor en CAR o CTD tienen un trato continuo con sus deportistas. La presencia del entrenador en las competiciones se hace aún más complicada, pues implica un gran esfuerzo económico. Recordemos que estos atletas han de lograr una buena clasificación en el ranking mundial, para ello compiten en la Copa del Mundo que se disputa a lo largo de toda la geografía mundial, así como en Campeonatos del Mundo y de Europa. La clase media, a la que pertenecen la mayoría de los entrenadores, y el hecho de compaginar dos trabajos, apoya firmemente esta tesis.

En todo este contexto, el ambiente del E.A.N. con sus triatletas es muy bueno, algo imprescindible para el entendimiento diario. Podemos decir también, que el entorno familiar es favorecedor de la labor de los entrenadores, pues, en casi la mitad de los casos, la práctica del triatlón o alguna de sus disciplinas individuales está presente. Sin duda la vocación deportiva del entrenador es clara, pues no entrena por dinero y sus estudios y trabajos se encuentran vinculados con el mundo deportivo.

Atendiendo al segundo punto de los resultados, los objetivos a medio y largo plazo marcados por los entrenadores se centran mayoritariamente en competición internacional, lo cual viene avalado por el nivel de la muestra. Algunos de ellos muestran su interés en atender las categorías de menores, dada la importancia que pueden tener para el futuro de este deporte.

A diferencia de otros deportes, el triatlón de alto nivel no se desarrolla bajo una estructura de clubes, aunque se pueda nutrir de ellos (Ruiz, 2006), pues los deportistas no requieren necesariamente a los mismos compañeros de entrenamiento, como sucede en los deportes de equipo, ni una instalación específica y única para un grupo estable de personas, como ocurre en los deportes de combate o sincronizados (Salinero y Ruiz, 2010). No obstante, los entrenadores de la muestra mantienen buenas relaciones con sus clubes e instituciones relacionadas.

$\mathrm{Al}$ exponer los resultados relacionados con el nivel de estudios de los entrenadores, se obtuvo casi un $80 \%$ de licenciados en Ciencias de la Actividad Física y el Deporte (CAFYD). Consideramos este dato como muy positivo al suponer una amplia formación directamente relacionada con la profesión de entrenador. Si comparamos esta cifra con otros estudios, la lectura es aún más positiva. En un estudio (M. C. Pérez, 2001) con 11 entrenadores hombres de alto rendimiento de tres deportes diferentes (cinco de fútbol, tres de tenis y tres de atletismo), sólo uno (9\%) poseía la licenciatura en CAFYD. En otro estudio reciente llevado a cabo con 17 entrenadores de alto nivel de kárate, todos ellos hombres, se obtuvo una cifra de 11,7\% de licenciados en CAFD (Salinero y Ruiz, 2010).

Retomando el segundo objetivo del estudio, en referencia a las cualidades atribuibles a un buen E.A.N. sabemos que, bajo la opinión de los propios entrenadores, la confianza para el diálogo personal se antepone a todos los demás. Esta sobredimensión del plano social sobre el deportivo, ha sido atribuida al entrenador experto, sobre todo en periodos de formación (Orlick, 1999; Salmela, 1995). Y es precisamente este aspecto de formación el que preocupa a los entrenadores, a pesar de encontrarse en el ámbito del alto nivel. Así lo demostraban cuando dentro de sus objetivos a medio y largo plazo, aparecía un especial interés por potenciar la base desde las categorías de menores. En deportes de equipo, los vínculos afectivos han sido también un factor identificado como cualidad del entrenador experto en la última etapa deportiva, de perfeccionamiento, según la clasificación de Bloom (1985). En otro orden de importancia aparecen igualados los conocimientos de entrenamiento y la capacidad para motivar y animar. Los entrenadores en triatlón han de manejar un cuádruple entrenamiento (natación, ciclismo, carrera a pie y preparación en gimnasio), teniendo en 
Ruiz, G.; Salinero, J. J. (2011) El entrenador de alto nivel en triatlón: entorno próximo y cualidades fundamentales para el rendimiento. Revista Internacional de Ciencias del Deporte, 23(7), 113-125. http://www.cafyd.com/REVISTA/02304.pdf

cuenta las interacciones positivas y negativas entre unas y otras disciplinas para integrarlo todo en un solo deporte. Por tanto no es de extrañar que esta cualidad sea valorada muy positivamente, junto con la capacidad para motivar y animar. La motivación y el refuerzo se convierten así en el alimento diario del triatleta de alto nivel que se enfrenta cada día, deliberadamente, al reto de la superación y la excelencia.

Por su parte, el factor competencia en las destrezas (dominio técnico) se encuentra entre los factores menos valorados. Para la etapa de alto nivel, el dominio técnico del entrenador no es considerada una cualidad importante, al igual que ocurre con los deportes de equipo (G. A. Bloom y Salmela, 2000). En todo caso, la presencia del entrenador como fuente de feedback técnico tiene un mayor sentido en los entrenamientos de natación, la disciplina más técnica. En este caso, el entrenador puede ejercer un mayor control favorecido por las limitaciones del espacio, no ocurriría así en las otras dos disciplinas de recorridos más abiertos (carrera y ciclismo).

\section{Conclusiones}

El triatlón de alto nivel en España se rige por un sistema centralizado en Centros de Alto Rendimiento y Centros de Tecnificación Deportiva. Los entrenadores que desempeñan su labor en estos centros son los que más triatletas de nivel internacional dirigen.

El binomio entrenador-deportista es la parte central y resultante del microsistema deportivo. Entre ambos el trato es excelente, en un deporte en el que la presencia del entrenador en competiciones y entrenamientos no es determinante para obtener un buen rendimiento en el alto nivel. Se considera aceptable el ratio de triatletas de alto nivel por entrenador de 3,7/1.

El ambiente en el microsistema se puede catalogar como bueno, dado el grado de satisfacción y trato del entrenador con los agentes del entorno (triatletas, otros entrenadores, federaciones y club). En el entorno familiar, menos de un tercio de la muestra posee familiares directos que practican triatlón o algunas de sus disciplinas individuales (natación, ciclismo, atletismo).

Los entrenadores de alto nivel españoles poseen un nivel académico alto y específico a la profesión que ejercen, siendo casi el 80\% de los mismos Licenciados en Ciencias de la Actividad Física y del Deporte. El estatus socioeconómico familiar estimado los sitúa principalmente en la clase media.

Entre los factores más valorados por parte de los entrenadores a la hora de determinar su labor, son los conocimientos de entrenamiento y la capacidad para motivar y animar. Sin embargo, por encima de estos dos factores aparece la confianza para el diálogo personal como el más valorado. El entorno del entrenador es en todo caso favorecedor del rendimiento experto, dada su alta implicación en un deporte no profesionalizado, donde ha de compaginar su labor de entrenador con otro trabajo, habitualmente también relacionado con el deporte. Sus estudios y entorno familiar se encuentran mayoritariamente también dentro del ámbito del deporte.

Los resultados que arroja esta investigación sacan a la luz la forma en que los entrenadores valoran su entorno de rendimiento. Es necesario que los propios deportistas y directivos asimilen estos datos para mejorar, si cabe, el entendimiento mutuo y tomar decisiones sobre aspectos tan importantes como la presencia de los entrenadores, o el número de deportistas/entrenador, para unificar un camino hacia la excelencia.

En futuras investigaciones deben contrastarse las valoraciones de los entrenadores con las de sus propios deportistas y ampliar las referencias de aquellos en cuanto a su experiencia deportiva y estrategias de planificación. 
Ruiz, G.; Salinero, J. J. (2011) El entrenador de alto nivel en triatlón: entorno próximo y cualidades fundamentales para el rendimiento. Revista Internacional de Ciencias del Deporte, 23(7), 113-125. http://www.cafyd.com/REVISTA/02304.pdf

\section{Referencias bibliográficas}

Alvira, F. (2004). La encuesta: una perspectiva general metodológica. Madrid: Centro de Investigaciones Sociológicas.

Baker, J., y Deakin, J. M. (2003). Training characteristics of expert and non-expert ultraendurance triathletes. Trabajo presentado en NASPSPA Conference, Savannah, Ga. Journal of Sport and Exercise Psychology, 25(Supl.).

Banister, E. W.; Carter, J. B., y Zarkadas, P. C. (1999). Training theory and taper: validation in triathlon athletes. European Journal of Applied Physiology, 79(2), 182191.

Bertalanffy, L. V. (1968). General System Theory: Foundations, Developmen, Applications. New York: George Braziller.

Bloom, B. S. (1985). Developing talent in youg people. New York: Ballantine.

Bloom, G. A.; Durand-Bush, N.; Schinke, R. J., y Salmela, J. H. (1998). The importance of mentoring in the development of coaches and athletes. International Journal of Sport Psychology, 29(3), 367-281.

Bloom, G. A., y Salmela, J. H. (2000). Personal characteristics of expert team sport coaches. Journal of Sport Pedagogy, 29(3), 56-76.

Bloom, G. A.; Schinke, R. J., y Salmela, J. H. (1998). The development of communication skills by elite basketball coaches. Coaching and Sport Science Journal, 2(3), 3-10.

British Triathlon. (2011). Clubmark Criteria: The Coaching and Competition Programme. Extraído

de http: // www. britishtriathlon.org/clubs/page. php?article=386\&category=/clubs $/ \&$ folder $=$ clubstandards $/ \&$ sub=clubmark $/$

Broom, E. F. (1981). Meeting the educational and sporting needs of the elite young athlete: A comparison of national organizational models. Comparative Physical Educationan and Sport, 8(3), 11.

Cavalheiro, C. A.; Soter, P. C., y Gagliardi, P. C. (2005). Multidisciplinary Training: The Orbital Model. New Studies in Athletics, 20(4), 7-18.

Cea, M. A. (1999). Metodología cuantitativa : estrategias y técnicas de investigación social. Madrid: Síntesis.

Consejo Superior de Deportes. Consultado Diciembre-2005, de http: //www.csd. mec. es/altacompeticion/ccaa/fede.htm

Côte, J., y Salmela, J. H. (1996). The organizational tasks of high performance gymnastic coaches. The Sport Psychology, 10(3), 247-260.

Côte, J.; Salmela, J. H., y Russell, S. J. (1995). The knowledge of high performance gymnastic coaches: Competition and training considerations. The Sport Psychology, 9, 76-95.

Chang, M. G., y Johnson, L. W. (1995). Segmenting the triathlon association membership market: an australian example. Sport Marketing Quarterly, 4(4), 25-28.

De Bosscher, V., y De Knop, P. (2002). The influence of sorts policies on international success: An international comparative study. Trabajo presentado en Sport for All and Elite Sport: Rivals or partners? $9^{\text {th }}$ World Sport for All Congress, Ahrnem (Ned).

Díaz, V.; Zapico, A.; Peinado, A.; Álvarez, M.; Benito, P., y Calderón, F. (2009). Physiological profile of elite triathletes: a comparison between young and professional competitors. Journal of Human Sport \& Exercise, 4(3), 237-246. 
Ruiz, G.; Salinero, J. J. (2011) El entrenador de alto nivel en triatlón: entorno próximo y cualidades fundamentales para el rendimiento. Revista Internacional de Ciencias del Deporte, 23(7), 113-125. http://www.cafyd.com/REVISTA/02304.pdf

Digel, H. (2002). A comparison of competitive sport systems. New Studies in Athletics, 17(1), 37-49.

Farber, H., Arbetter, J., Schaefer, E., Hill, S., Dallal, G., Grimaldi, R. y Hill, N. (1987). Acute metabolic effects of an endurance triathlon. Annals of Sport Medicine, 3(2), 131-138.

García-Ferrando, M. (1996). Los deportistas olímpicos españoles: Un perfil sociológico. Madrid: Consejo Superior de Deportes.

García-Ferrando, M. (2003). La encuesta El análisis de la realidad social. Métodos y técnicas de investigación. Madrid: Alianza.

García, M.; I báñez, J., y Alvira, F. (2002). El análisis de la realidad social. Métodos y técnicas de investigación ( $3^{a}$ ed.). Madrid: Alianza.

Haslam, I. R., y Smith, D. E. (2001). An investigation of the coaching behaviours among Singapore coaches. Journal of Physical Education and Recreation, 7(1), 21-25.

Hill, R.; McConnell, A.; Forster, T., y Moore, J. (2002). A comprehensive view of development of U.S. Olympians who competed from 1984-1998. Initial report: Results of the talent identification and development quiestionnaire to U.S. Olympians. : Athlete development and Coaching \& Sport Sciences divisions.

Hilliard, D. C. (1986). The Staging of Emotions in Endurance Athletic Competitions: Implications for Successful Race Management. Trabajo presentado en Annual Meetings of the Western Social Science Association, Reno, Nevada.

Hue, O.; Valluet, A.; Blonc, S., y Hertogh, C. (2002). Effects of multicycle-run trainning on triathlete performance. Research Quarterly for Exercise and Sport, 73(3), 289295.

Institut für Angewandte Trainingswissenschaft of Leipzig. (2006). Base estadística de resultados internacionales en triatlón. Consultado 2006, de http://www.iat.unileipzig. de/datenbanken/dbtriathlon/start.php

Isaksen, S. G. (1998). A review of brainstorming research: six critical issues for inquiry. New York: Creative Problem Solving Group - Buffalo ${ }^{\circledR}$.

Kohrt, W. M.; Morgan, D. W.; Bates, B., y Skinner, J. S. (1987). Physiological responses of triathletes to maximal swimming, cycling and running. Medicine and Science in Sports and Exercise, 19(1), 51-55.

Kreider, R., Boone, T., Thompson, W., Burkes, S. y Cortes, C. (1988). Cardiovascular and thermal responses of triathlon performance. Medicine and Science in Sports and Exercise, 20(4), 385-390.

Laursen, P. B., y Rhodes, E.C. (2001). Factors affecting performance in an ultraendurance triathlon. Sports Medicine, 31(3), 195-209.

Likert, R. (1932). A technique for the measurement of attitudes. Archives of Psychology, 22(140), 1-55.

Miura, H., Kitagawa, K. y Ishiko, T. (1999). Characteristic feature of oxygen cost at simulated laboratory triathlon test in trainded triathletes. Journal of Sports Medicine and Physical Fitness, 39(2), 101-106.

O'Toole, M. L. (1989). Training for ultraendurance triathlons. Medicine and Science in Sports and Exercise, 21(5), 209-213.

Orlick, T. (1999). Promover a confiança a alegría e a excelencia no tremo de jovens. Seminario Internacional Tremo de Jovens, los caminhos do sucesso. Lisboa: Centro de Estudos e Formaçao Desportiva.

Osborn, A. F. (1963). Applied imagination (3a ed.). New York: Scribner. 
Ruiz, G.; Salinero, J. J. (2011) El entrenador de alto nivel en triatlón: entorno próximo y cualidades fundamentales para el rendimiento. Revista Internacional de Ciencias del Deporte, 23(7), 113-125. http://www.cafyd.com/REVISTA/02304.pdf

Pérez, J. (1977). Encuesta a atletas de élite. Tesina sin publicar, Escuela Superior de Educación Física, Madrid.

Pérez, M. C. (2001). Estudio cualitativo sobre entrenadores de alto rendimiento deportivo. Revista de Psicología del Deporte, 11(1), 9-33.

Rossi, B. (1977). Método Punto-Deporte. Cuaderno dello Sport, año 1977.

Ruiz, G. (2006). El triathlon español de alto nivel: análisis cualitativo y cuantitativo. Trabajo presentado en IX J ornadas Técnicas de Triathlon, Madrid.

Ruiz, G.; Salinero, J. J., y Sánchez-Bañuelos, F. (2008). Valoración del perfil sociodemográfico en el triatleta: el ejemplo de Castilla-La Mancha. Nivel de implicación y entorno. Apunts, 92(2), 5-14.

Sage, G. H. (1980). Socialization of coaches: Antecedents to coaches' beliefs and behaviors Sport and American Society: Selected Readings (pp. 160-170). Wesley: Addison.

Salinero, J. J., y Ruiz, G. (2010). El entrenador de alto rendimiento deportivo y su contraste con entrenadores de menor nivel. Estudio en la modalidad de karate en España. Motricidad. European J ournal of Human Movement, 24, 107-119.

Salmela, J. H. (1995). Learning from the development of expert coaches. Coaching and Sport Science Journal, 2(2), 3-13.

Sánchez-Bañuelos, F. (2002). Factores Psicosociales Clave para la Selección de Talentos Deportivos: El diseño de un programa de seguimiento para deportistas jóvenes con posibilidades de acceso al Plan Regional "Castilla-La Mancha Olímpica" (CLAMO). Toledo: Consejería de Deportes de la Junta de Comunidades de Castilla-La Mancha.

Sleivert, G. G., y Rowlands, D. S. (1996). Physical and physiological factors associated with success in the triathlon. Sports Medicine, 22(1), 8-18.

Sleivert, G. G., y Wenger, H. A. (1993). Physiological predictors of short-course triathlon performance. Medicine and Science in Sports and Exercise, 25(7), 871-876.

Tatano, H. (1977). A framework for a Sport System. Research of Physical Education, $5(5), 65-81$.

Varela, C. (2003). Planificación y entrenamiento en el alto nivel. Trabajo presentado en el XI Curso de Entrenador Superior de Triatlón, Madrid.

Zhou, S.; Robson, S. J.; King, M. J., y Davie, A. J. (1997). Correlations between shortcourse triathlon performance and physiological variables determined in laboratory cycle and treadmill tests. Journal of Sports Medicine and Physical Fitness, 37(12), 122-130.

Agradecimientos: A la Federación Española de Triatlón. 